\title{
Short communication: Methicillin-resistant Staphylococcus aureus detection in US bulk tank milk
}

\author{
J. E. Virgin, ${ }^{*}$ T. M. Van Slyke,† J. E. Lombard, ${ }^{* 1}$ and R. N. Zadokst ${ }^{2}$ \\ *USDA:APHIS:VS, Centers for Epidemiology and Animal Health, 2150 Centre Ave., Bldg B, Fort Collins, CO 80526-8117 \\ †Quality Milk Production Services, College of Veterinary Medicine, Cornell University, Ithaca, NY14850-1263
}

\begin{abstract}
Staphylococcus aureus is a major cause of mastitis in dairy cattle. This study estimated the herd prevalence of methicillin-resistant Staph. aureus (MRSA) among US dairy herds by testing bulk tank milk (BTM) samples using genotypic and phenotypic methods. A nationally representative sample of 542 operations had BTM cultured for Staph. aureus, and 218 BTM samples were positive upon initial culture. After 4 wk to 4 mo of frozen storage, $87 \%$ of 218 samples $(\mathrm{n}=190)$ were still culture positive for Staph. aureus on blood agar, but none were positive for MRSA on the selective indicator medium CHROMagar MRSA. A duplex PCR was used to detect the Staph. aureus-specific nuc gene and the methicillin resistance gene, mecA, in mixed staphylococcal isolates from the 190 BTM samples that were positive for Staph. aureus after storage. Seven samples tested positive for nuc and mecA, and 2 samples tested positive for mecA only. MecA-positive Staphylococcus spp., but not MRSA, were subsequently isolated from 5 samples, whereas neither mecA-positive Staphylococcus spp. nor MRSA was isolated from the remaining 4 samples. Presence of methicillin-resistant, coagulasenegative Staphylococcus spp. may complicate the detection of MRSA by means of PCR on BTM. Bulk tank milk in the United States is not a common source of MRSA.
\end{abstract}

Key words: methicillin-resistant Staphylococcus aureus (MRSA), dairy cow, bulk tank milk, NAHMS

Methicillin-resistant Staphylococcus aureus (MRSA) is a major cause of nosocomial infections in humans. In addition, community-acquired MRSA has now become a major concern (Otter and French, 2008). New evidence also suggests that domestic animals, including food animals, are capable of serving as reservoirs and

\footnotetext{
Received April 13, 2009.

Accepted June 30, 2009.

${ }^{1}$ Corresponding author: jason.e.lombard@aphis.usda.gov

${ }^{2}$ Current address: Royal (Dick) School of Veterinary Studies, The University of Edinburgh, Easter Bush Veterinary Centre, Roslin, Scotland, UK.
}

shedders of MRSA and that transmission between host species may be possible (Loo et al., 2007). Methicillinresistant Staph. aureus has been detected in the milk of cows with mastitis, dogs, cats, horses, pigs, sheep, goats, rabbits, chickens, and exotic species (Lee, 2003; Leonard and Markey, 2006). Recent studies have shown genetic similarity between MRSA isolates from food animals, including dairy cows, and those in humans, suggesting a mode of transmission between the 2 species (Juhasz-Kaszanyitzky et al., 2007; Moon et al., 2007). Infection of dairy cattle with MRSA has been attributed to human-to-animal transfer, but the directionality of transmission is not always known (Devriese and Hommez, 1975; Juhasz-Kaszanyitzky et al., 2007). Although Staph. aureus is a common mastitis pathogen, there has been little evidence to suggest that MRSA is common in milk. Furthermore, the risk of dairy product-borne transmission of MRSA is easily minimized by pasteurization, and most reported instances of foodborne MRSA have occurred through contamination by infected food handlers rather than the food itself (Kluytmans et al., 1995; Jones et al., 2002).

Previous research indicates that MRSA is rarely found in milk from dairy cattle in the United States. Analysis of 2,978 Staph. aureus isolates from milk samples submitted to diagnostic laboratories in Michigan and Wisconsin between 1994 and 2001 showed that 1.4\% of isolates were resistant to oxacillin (Erskine et al., 2002; Makovec and Ruegg, 2003). Resistance to oxacillin was not detected among 357 Staph. aureus isolates recovered from milk in North Carolina (Anderson et al., 2006). A survey of milk samples from cows with mastitis from 153 dairies in Korea, however, yielded $21(2.5 \%)$ MRSA positives and 19 (2.4\%) methicillinresistant CNS (Moon et al., 2007). Further studies are needed in the United States to ascertain the prevalence of MRSA in food animals, especially dairy cattle. The objective of this study was to determine the prevalence of MRSA detected in bulk tank milk (BTM) samples that were collected as part of the National Animal Health Monitoring System's (NAHMS) Dairy 2007 study and represented the diversity of dairy operations in the United States. Bulk tank milk samples were sub- 
mitted to Quality Milk Production Services (QMPS, Ithaca, NY) for detection of mastitis pathogens and MRSA. We hypothesized that Staphylococcus aureus would be isolated from BTM samples but the prevalence of MRSA would be low.

The NAHMS Dairy 2007 study was conducted in 17 of the top US dairy-producing states. The regions (states) included West (California, Idaho, New Mexico, Texas, and Washington) and East (Indiana, Iowa, Kentucky, Michigan, Minnesota, Missouri, New York, Ohio, Pennsylvania, Vermont, Virginia, and Wisconsin). Herds were categorized by the number of milk cows into small (1-99 cows), medium (100-499 cows), and large $(500+$ cows $)$. Phase I of the study covered the population of dairy operations with at least one milk cow in the 17 participating states. As of January 1,2007 , these operations accounted for $82.5 \%$ of milk cows and $79.5 \%$ of dairy operations in the United States. Collection of BTM occurred during phase II of the study and included those operations in phase I that elected to continue participating and had at least 30 milk cows. All respondent data were statistically weighted to reflect the population of dairy operations with 30 or more cows in the 17 participating states. The inverse of the probability of selection for each operation was the initial selection weight. The selection weight was adjusted for nonresponse within each state and size group to allow for inferences back to the original sample population. Weights were again adjusted to account for nonrespondents in phase II. Of the 3,554 operations in the original sample, 2,194 completed phase I and of the 1,073 eligible operations, 582 completed phase II. Between February 28 and August 30, 2007, approximately $100 \mathrm{~mL}$ of bulk tank milk was aseptically collected from 542 of the 582 operations that completed phase II. Samples were packed in ice and shipped overnight to QMPS.

The BTM samples were received by the QMPS bacteriology laboratory and cultured for the presence of mastitis pathogens including Staph. aureus using routine methods (Hogan et al., 1999; Zadoks et al., 2004). The samples then were stored at $-20^{\circ} \mathrm{C}$ for 4 wk to 4 mo until further processing. To detect MRSA in BTM, phenotypic and genotypic methods were used. Phenotypic detection was based on plating on a selective indicator medium, BBL CHROMagar MRSA (BD Diagnostic Systems, Sparks, MD; Han et al., 2007). Samples that tested positive for Staph. aureus upon initial routine culture $(\mathrm{n}=218)$ were thawed at room temperature and streaked onto CHROMagar MRSA using sterile cotton swabs. As positive controls, MRSA strain ATCC 700698 and strain QMP S1-027, a MRSA isolate obtained from heifer milk and confirmed by nuc and mecA PCR (gift of John Barlow, University of Ver- mont, Burlington), were used. Plates were incubated aerobically at 35 to $37^{\circ} \mathrm{C}$ and evaluated after $24 \pm 4$ h. Presence and appearance of colonies were recorded, and special attention was paid to colony color. Mauve colonies on CHROMagar MRSA were observed for both control strains, but not for any of the BTM samples tested. Blue colonies were observed on a large proportion of CHROMagar MRSA plates $(\mathrm{n}=145$, or $67 \%$ of BTM samples tested), and pale pink colonies or blue colonies with a pink halo were observed on 11 plates $(5 \%$ of samples tested). Based on microscopic evaluation of colony morphology, catalase and coagulase testing, all pink colonies, all blue colonies with a pink halo, and an arbitrarily selected subset of blue colonies were identified to the genus level. Colonies belonged to the genera Streptococcus (blue colonies with or without pink halo), Corynebacterium (pale pink colonies), or Staphylococcus (pale pink colonies; all coagulase negative). A large proportion of BTM samples contain Streptococcus spp., Staphylococcus spp., and Corynebacterium spp. (Zadoks et al., 2004). Staphylococcus and Corynebacterium species are known to give occasional false-positive results on CHROMagar MRSA (Han et al., 2007). Streptococcus spp., Staphylococcus spp., and Corynebacterium spp. were phenotypically distinct from MRSA isolates in the current study, but growth of such colonies on CHROMagar MRSA complicates interpretation of culture results and may lead to false-positive readings. Thus, CHROMagar MRSA, which was developed for detection of MRSA in nasal swabs, may not be suitable for routine detection of MRSA in BTM.

In a parallel assay, the thawed milk samples were plated on trypticase soy agar with $5 \%$ sheep blood and 0.1 esculin (TSA-BE; PML Microbiologicals, Mississauga, Ontario, Canada) to obtain staphylococcal colonies for subsequent genotypic detection of MRSA based on PCR using Staph. aureus and mecA-specific primer sets (Brakstad et al., 1992; Martineau et al., 2000). A mixture of staphylococcal colonies was used to represent the mixed staphylococcal flora that can be present in bulk milk samples. For samples that tested positive for nuc and mecA, PCR was repeated on subcultures of isolated staphylococcal colonies to determine whether the 2 genes were present in the same isolate or in different isolates. This stepwise approach enabled us to process a large number of samples at low cost. For screening of the BTM samples, mixed staphylococcal colonies from TSA-BE were suspended in $1 \times$ Tris-EDTA buffer (Promega, Madison, WI). An aliquot of the suspension was used as DNA template in PCR reactions and the remainder was stored at $4^{\circ} \mathrm{C}$. The $n u c$ primers $(\mathrm{NUC} 1=$ 5'-GCG ATT GAT GGT GAT ACG GTT-3' and NUC2 $=5^{\prime}$-AGC CAA GCC TTG ACG AAC TAA AGC- $3^{\prime}$ ) were used to detect the gene encoding the thermostable 
nuclease of Staph. aureus (Brakstad et al., 1992). The $m e c A$ primers $\left(\right.$ mecA174 $=5^{\prime}$-AAC AGG TGA ATT ATT AGC ACT TGT AAG-3' and mecA174B $=5^{\prime}$ ATT GCT GTT AAT ATT TTT TGA GTT GAA$3^{\prime}$ ) were used to detect the methicillin resistance gene (Martineau et al., 2000). Reactions were run in a total volume of $25 \mu \mathrm{L}$ consisting of $2 \mu \mathrm{L}$ of template, $12.5 \mu \mathrm{L}$ of GoTaq Green (Promega), $0.125 \mu \mathrm{L}$ of each primer $(100 \mathrm{mM})$, and $10 \mu \mathrm{L}$ of nuclease-free $\mathrm{dH}_{2} \mathrm{O}$, using an iCycler (BioRad Laboratories, Hercules, CA) and the following run parameters: initial denaturation at $94^{\circ} \mathrm{C}$ for $15 \mathrm{~min}$, followed by 37 cycles at $94^{\circ} \mathrm{C}$ for $60 \mathrm{~s}, 55^{\circ} \mathrm{C}$ for $30 \mathrm{~s}$, and $72^{\circ} \mathrm{C}$ for $60 \mathrm{~s}$, and final extension at $72^{\circ} \mathrm{C}$ for $10 \mathrm{~min}$. In each PCR assay, the 2 MRSA control strains and 2 negative controls were included, one for lysate preparation and one for the PCR reaction itself. Presence of nuc and mecA PCR products, which are 270 and $174 \mathrm{bp}$ long, respectively, was determined through electrophoresis in $1.5 \%$ agarose gels. Of the 218 BTM samples that had been positive for Staph. aureus upon initial culture (i.e., before frozen storage of the milk samples), 190 (87.2\%) tested positive for Staph. aureus using the PCR method on a mixture of staphylococcal colonies from the poststorage culture. The failure to detect Staph. aureus in $28(12.8 \%)$ of samples after frozen storage may be the result of loss of viability during storage, random variability in detection of Staph. aureus in samples with low numbers of colony-forming units, or lack of sensitivity of the culture and PCRbased methods used after frozen storage.

Nine colony suspensions tested positive for $m e c A$. In 7 samples, nuc and mecA were detected, and in 2 samples $m e c A$ but not nuc was detected. Detection of mecA in the absence of nuc can be explained by the presence of methicillin-resistant Staphylococcus spp. other than Staph. aureus. Methicillin resistance is more common in non-aureus staphylococci from milk samples than in Staph. aureus (Makovec and Ruegg, 2003). Given that detection of mecA can be due to presence of Staphylococcus spp., there was a need to determine whether nucpositive, mecA-positive samples contained MRSA or a mixture of methicillin-resistant Staphylococcus spp. and methicillin-susceptible Staph. aureus (MSSA). To this end, the stored portion of the colony suspensions that had been used to set up PCR reactions was plated on TSA-BE and incubated overnight at $37^{\circ} \mathrm{C}$ for samples that had yielded mecA amplicons. After incubation, individual staphylococcal colonies were streaked for isolation onto fresh TSA-BE to obtain pure cultures, which were subsequently tested by nuc-mecA PCR to determine whether MSSA, MRSA, or methicillinresistant Staphylococcus spp. had been present in the suspension. Methicillin-resistant Staphylococcus spp. but not MRSA were obtained from 5 BTM samples. No $m e c A$-positive colonies were isolated from the remaining 4 samples. One of those samples had tested negative for nuc, suggesting that methicillin-resistant Staphylococcus spp. was the source of the mecA gene. For the remaining 3 samples, we could not determine whether the mecA gene originated from Staphylococcus spp. or Staph. aureus. Testing of isolated rather than mixed colonies from BTM samples would preclude the need to determine whether mecA-positive results were due to MRSA or Staphylococcus spp. Given the high prevalence of Staph. aureus in BTM and the low prevalence of mecA, such an approach might be cost-prohibitive in routine veterinary diagnostic laboratories.

On the basis of our results we conclude that 1) MRSA could not be detected in nationally representative BTM samples from the NAHMS Dairy 2007 study using phenotypic (CHOMagar MRSA) and genotypic (nuc-mecA PCR) methods, suggesting that BTM is not a common source of MRSA in the United States; 2) the mecA gene that encodes methicillin resistance does occur in staphylococci isolated from BTM; 3) presence of mecA in BTM isolates often is due to presence of methicillinresistant Staphylococcus spp. other than Staph. aureus; and 4) there is a need for validated, cheap, and fast tools to detect MRSA in BTM routinely and to differentiate it from other methicillin-resistant Staphylococcus spp. that may be present in BTM. Such a tool could contribute to routine monitoring of milk quality and safety.

\section{REFERENCES}

Anderson, K. L., R. L. Lyman, S. M. Bodeis-Jones, and D. G. White. 2006. Genetic diversity and antimicrobial susceptibility profiles among mastitis-causing Staphylococcus aureus isolated from bovine milk samples. Am. J. Vet. Res. 67:1185-1191.

Brakstad, O. G., K. Aasbakk, and J. A. Maeland. 1992. Detection of Staphylococcus aureus by polymerase chain reaction amplification of the nuc gene. J. Clin. Microbiol. 30:1654-1660.

Devriese, L. A., and J. Hommez. 1975. Epidemiology of methicillinresistant Staphylococcus aureus in dairy herds. Res. Vet. Sci 19:23-27.

Erskine, R. J., R. D. Walker, C. A. Bolin, P. C. Bartlett, and D. G. White. 2002. Trends in antibacterial susceptibility of mastitis pathogens during a seven-year period. J. Dairy Sci. 85:11111118.

Han, Z., E. Lautenbach, N. Fishman, and I. Nachamkin. 2007 Evaluation of mannitol salt agar, CHROMagar Staph aureus and CHROMagar MRSA for detection of methicillin-resistant Staphylococcus aureus from nasal swab specimens. J. Med. Microbiol. 56:43-46.

Hogan, J. S., R. N. González, R. J. Harmon, S. C. Nickerson, S. P. Oliver, J. W. Pankey, and K. L. Smith. 1999. Laboratory Handbook on Bovine Mastitis. Rev. ed. National Mastitis Council, Madison, WI.

Jones, T. F., M. Kellum, S. Porter, M. Bell, and W. Schaffner. 2002. An outbreak of community-acquired foodborne illness caused by methicillin-resistant Staphylococcus aureus. Emerg. Infect. Dis. $8: 82-84$.

Juhasz-Kaszanyitzky, E., S. Janosi, P. Somogyi, A. Dan, L. Van der Graaf-van Bloois, E. Van Duijkeren, and J. A. Wagenaar. 2007. 
MRSA transmission between cows and humans. Emerg. Infect. Dis. 13:630-632.

Kluytmans, J., W. van Leeuwen, W. Goessens, R. Hollis, S. Messer, L. Herwaldt, H. Bruining, M. Heck, J. Rost, N. van Leeuwen, A. Van Belkum, and H. Verbrugh. 1995. Food-initiated outbreak of methicillin resistant Staphylococcus aureus analyzed by pheno- and genotyping. J. Clin. Microbiol. 33:1121-1128.

Lee, J. H. 2003. Methicillin (oxacillin)-resistant Staphylococcus aureus strains isolated from major food animals and their potential transmission to humans. Appl. Environ. Microbiol. 69:64896494.

Leonard, F. C., and B. K. Markey. 2006. Methicillin-resistant Staphylococcus aureus in animals: A review. Vet. J. 175:17-36.

Loo, I., X. Huijsdens, E. Tiermersma, A. Neeling, N. van de SandeBruinsma, D. Beaujean, A. Voss, and J. Klutmans. 2007. Emergence of methicillin-resistant Staphylococcus aureus of animal origin in humans. Emerg. Infect. Dis. 13:1834-1839.

Makovec, J. A., and P. L. Ruegg. 2003. Antimicrobial resistance of bacteria isolated from dairy cow milk samples submitted for bacterial culture: 8,905 samples (1994-2001). J. Am. Vet. Med. Assoc. 222:1582-1589.
Martineau, F., F. J. Picard, N. Lansac, C. Menard, P. H. Roy, M. Ouellette, and M. G. Bergeron. 2000. Correlation between the resistance genotype determined by multiplex PCR assays and the antibiotic susceptibility patterns of Staphylococcus aureus and Staphylococcus epidermidis. Antimicrob. Agents Chemother. 44:231-238.

Moon, J. S., A. R. Lee, H. M. Kang, E. S. Lee, M. N. Kim, Y. H. Paik, Y. H. Park, Y. S. Joo, and H. C. Koo. 2007. Phenotypic and genetic antibiogram of methicillin-resistant Staphylococci isolated from bovine mastitis in Korea. J. Dairy Sci. 90:1176-1185.

Otter, J. A., and G. L. French. 2008. The emergence of communityassociated methicillin-resistant Staphylococcus aureus at a London teaching hospital, 2000-2006. Clin. Microbiol. Infect. 14:670676 .

Zadoks, R. N., R. N. González, K. J. Boor, and Y. H. Schukken. 2004. Mastitis-causing streptococci are important contributors to bacterial counts in raw bulk tank milk. J. Food Prot. 67:26442650 . 